\title{
Throughput optimality of delay-driven MaxWeight scheduler for a wireless system with flow dynamics
}

\author{
Bilal Sadiq and Gustavo de Veciana \\ Dept. of Electrical and Computer Engineering \\ The University of Texas at Austin
}

\begin{abstract}
We consider a wireless downlink shared by a dynamic population of flows. The flows of random size (bits) arrive at the base station at random times, and leave when they have been completely transmitted. The transmission rate supported by the wireless channel of each flow while the flow awaits transmission varies randomly over time and is independent of that of the other flows. The scheduling problem in this context is to select a flow for transmission based on the current system state (e.g., backlogs, wait times, and channel states of the contending flows). It has recently been shown that for such a system, the wellknown (backlog-driven) MaxWeight scheduler is not throughput optimal. That is to say, the MaxWeight scheduler will not stabilize a given system even though it is possible to construct a stabilizing scheduler using the various flow- and channel-related statistics. However, in this paper, we show that the delay-driven MaxWeight scheduler is, nevertheless, throughput optimal for such a system. The delay-driven MaxWeight, like its backlog-driven version, does not require any knowledge of the flow- or channel-related statistics.
\end{abstract}

\section{INTRODUCTION}

The time-varying nature of wireless channels provides an opportunity to schedule the flows/users when they see a favorable channel states - this is referred to as opportunistic scheduling [1]-[3]. In a dynamic system, i.e., one where users' data or even new users arrive into the system as a random process, the opportunistic or channel-aware schedulers may not be stable, i.e., keep the data/user queues bounded, unless they are carefully designed, e.g., possibly using prior knowledge of the arrival and channel processes [4], [5].

For systems with time-varying channels but a fixed number of users whose data packets arrive as a stationary random process, there are well-known queue- and channel- aware schedulers that are provably throughput optimal in a variety of network settings. A scheduler in this context is said to be throughput optimal, if without the knowledge of arrivals and channel statistics it is able to stabilize the system, if at all possible under some other scheduler. Examples of such schedulers are the Longest Connected Queue [6], MaxWeight [7], Exponential rule [8], and Log rule [9]; see [5] for more details. A typical application of these schedulers in, e.g., deciding downlink packet transmissions from a wireless base station, would be to (try to) achieve low packet delays of the order of few tens to few hundreds of milliseconds [10] [11],

This research was supported in part by grants AFOSR FA9550-07-1-0428 and NSF CNS-0721532. when there is a given fixed number of users/flows which might correspond to real-time voice/video sessions etc.

However for best effort flows, the relevant performance metrics are defined over longer time scales, i.e., the time scales of flow-level dynamics, e.g., file transfer delays or web browsing interactivity. Unlike a system where there is a fixed number of users/flows and each flow generates a stationary packet arrival process, in this setting the arrivals correspond to new flows and users, i.e., files to be transferred associated with different users, and thus the number of ongoing flows in the system is dynamic. Each flow can be viewed as having its own queue associated with the residual data that needs to be transmitted in order to successfully transfer a file or web page. In this context, [5] recently showed that the queue-driven MaxWeight scheduler is not throughput optimal ${ }^{1}$.

In this paper, we show that the delay-driven MaxWeight still is throughput optimal in the dynamic flow setting. The critical observation which explains why the queue-driven version is not throughput optimal but the delay-driven version is, is as follows. In the setting where there is a fixed number of flows, a linear relation can be established between the headof-line packet delay and the queue length of a flow (Little's law) as either one gets large. By contrast, in the setting with dynamic number of flows, while the head-of-line delay of an un-served flow will continue to increase, its queue length will not due to the finite size of flows. So, in the former setting, the queue-driven and the delay-driven versions of MaxWeight are equivalent in some sense, whereas, in the latter, the queue-driven version may perpetually fail to exploit good channel states of small queues (i.e. files with few residual bits) irrespective of how long these small files wait while the bigger newer files may get scheduled (because of their longer queue lengths) even when their channels are poor; see excellent illustrative examples in [5].

\section{SYSTEM MODEL}

Let random $\boldsymbol{A}(t) \in \mathbb{Z}_{+}$denote the number of files arriving in time slot $[t, t+1)$, these files will not be available for service until the next time slot. We assume $\boldsymbol{A}(\cdot)$ are i.i.d. with finite mean $\lambda \equiv \mathbb{E} \boldsymbol{A}(0)$. For $0<i \leq \boldsymbol{A}(t)$, let $\boldsymbol{B}_{i}(t)$ denote the file size in bits of the $i^{\text {th }}$ arriving file.

\footnotetext{
${ }^{1}$ Queue-driven Exponential rule and Log rule can similarly be shown to be not throughput optimal in the dynamic flow setting.
} 
We assume that $\boldsymbol{B}_{i}(t)$ are i.i.d. (across both $t$ and $i$ ), are bounded, and have mean $\beta \equiv \mathbb{E} \boldsymbol{B}_{1}(0)$. We will use bold face, e.g. $(\boldsymbol{A}(t), t=0,1, \cdots)$, to denote the random process and plain font, e.g. $(A(t), t=0,1, \ldots)$, to denote a realization of the process. Also, we will make a distinction between "increasing" and "strictly increasing" and between "positive" and "strictly positive" etc.

Let $\boldsymbol{Q}(t) \in \mathbb{Z}_{+}$denote the number of files present in the system at the end of time slot $[t-1, t)$. Then in the time slot $[t, t+1)$, at most one of the $\boldsymbol{Q}(t)$ files present in the system can be scheduled to receive service. For each file $0<i \leq \boldsymbol{Q}(t)$ present in the system at the end of time slot $[t-1, t)$, let

- $\left[\boldsymbol{T}_{i}(t), \boldsymbol{T}_{i}(t)+1\right)$ denote the time slot in which the $i^{t h}$ file arrived. We index the files in order of their arrival time, i.e., $\boldsymbol{T}_{1}(t) \leq \boldsymbol{T}_{2}(t) \leq \cdots \leq \boldsymbol{T}_{\boldsymbol{Q}(t)}(t)$. Let $\boldsymbol{T}(t) \equiv\left(\boldsymbol{T}_{i}(t), 1 \leq i \leq \boldsymbol{Q}(t)\right)$ and $\boldsymbol{W}(t) \equiv$ $\left(\boldsymbol{W}_{i}(t), 1 \leq i \leq \boldsymbol{Q}(t)\right)$, where $\quad \boldsymbol{W}_{i}(t) \equiv t-\boldsymbol{T}_{i}(t)>0$ denotes the current wait time of the $i^{t h}$ file. Also, let $\boldsymbol{W}(t) \equiv 0$ if $\boldsymbol{Q}(t)=0$.

- random $\boldsymbol{R}_{i}(t)$ denote the maximum number of bits that can be served/transmitted from file $i$, if it is scheduled in time slot $[t, t+1)$. We will sometimes refer to $\boldsymbol{R}_{i}(t)$ as the state of the $i^{t h}$ files channel. We assume that $\boldsymbol{R}_{i}(t)$ lies in a finite set $\left\{0,1, \cdots, r^{\max }\right\}$ and is i.i.d. (across both $t$ and $i$ ). Also, let $p_{0} \equiv \mathbb{P}\left(\boldsymbol{R}_{1}(0)>0\right)$ and $p \equiv \mathbb{P}\left(\boldsymbol{R}_{1}(0)=r^{\max }\right)>0$.

- $\boldsymbol{L}_{i}(t)>0$ denote the number of bits left in the $i^{t h}$ file at the end of slot $[t-1, t)$, or, equivalently, the number of bits available for transmission in time slot $[t, t+1)$. A file leaves the system once all its bits have been served/transmitted. Also let $\boldsymbol{L}(t) \equiv\left(\boldsymbol{L}_{i}(t), 1 \leq i \leq \boldsymbol{Q}(t)\right)$

See Fig. 1 for a graphical illustration of the system.

Let $\gamma \equiv \mathbb{E}\left[\frac{B_{1}(0)}{r^{\max }}\right]$ and $\rho \equiv \lambda \gamma$, i.e., the average amount of work (in number of slots) per slot that is entering the system if the files could always be served at $r^{\max }$ bits/slot. We assume that $\epsilon_{0} \equiv 1-\rho>0$ which is a sufficient condition for stabilizablility [5].

Remark 1: We have assumed that file size and channel state are i.i.d. across files, however, consider the following generalization. Suppose there are a fixed number $K$ of classes of files, where the class determines the distribution of the arrival and the file size processes, as well as the distribution of the channel seen by the file. Then for each class $k \in\{1,2, \cdots, K\}$, suppose a file in the $k^{\text {th }}$ class sees an i.i.d. channel that has the same distribution as $\boldsymbol{R}_{1}^{k}(0)$. So the distribution of channel state may differ across classes (as indicated by the superscript $k$ ), however, we restrict that for all $k, \mathbb{P}\left(\boldsymbol{R}_{1}^{k}(0)=r^{\max }\right)>0$ and $\mathbb{P}\left(\boldsymbol{R}_{1}^{k}(0)>r^{\max }\right)=0$. That is, the highest possible rate supported by the channel of any file of any class is $r^{\max }$. Using this $r^{\max }$, let $\lambda^{k}, \beta^{k}, \gamma^{k}$ and $\rho_{k}$ respectively denote the mean arrival rate, file size, work load per file, and total work load associated with the $k^{t h}$ class. Then, the results presented in this paper are applicable, without modification, to this more general multi-class system model, by appropriately defining $\lambda, \beta, \gamma, \rho$ and $p$ as follows:

$$
\begin{gathered}
\lambda=\sum_{k=1}^{K} \lambda_{k}, \quad \beta=\sum_{k=1}^{K} \frac{\lambda_{k}}{\lambda} \beta_{k}, \quad \gamma=\sum_{k=1}^{K} \frac{\lambda_{k}}{\lambda} \gamma_{k}, \\
\rho=\sum_{k=1}^{K} \rho_{k}, \quad p=\min _{1 \leq k \leq K} \mathbb{P}\left(\boldsymbol{R}_{1}^{k}(0)=r^{\max }\right) .
\end{gathered}
$$

\section{Delay-driven MaxWeight scheduler}

Definition 1: For any time slot $[t, t+1)$, when there are $Q(t)>0$ files present in the system (at the end of the last slot), and the corresponding wait time and channel state vectors are $W(t)=\left(W_{i}(t), 1 \leq i \leq Q(t)\right)$ and $R(t)=\left(R_{i}(t), 0 \leq i \leq Q(t)\right)$ respectively, then schedule for service a file $i^{*}(W(t), R(t))$ that satisfies,

$$
i^{*}(W(t), R(t)) \in \arg \max _{1 \leq i \leq Q(t)} W_{i}(t) R_{i}(t),
$$

with ties broken in favor of the smallest index $i$ achieving the max in above.

Remark 2: Under MaxWeight, the process $\boldsymbol{Q}(\cdot)$ evolves as follows,

$$
\boldsymbol{Q}(t+1)=\left(\boldsymbol{Q}(t)-\mathbb{1}_{\left\{\boldsymbol{L}_{*}(t) \leq \boldsymbol{R}_{*}(t)\right\}}\right)^{+}+\boldsymbol{A}(t) .
$$

where $\quad \boldsymbol{L}_{*}(\cdot) \quad \equiv \quad \boldsymbol{L}_{i^{*}(\boldsymbol{W}(\cdot), \boldsymbol{R}(\cdot))}(\cdot) \quad$ and $\boldsymbol{R}_{*}(\cdot) \equiv \boldsymbol{R}_{i^{*}(\boldsymbol{W}(\cdot), \boldsymbol{R}(\cdot))}(\cdot)$. For later use, let us also define $\boldsymbol{T}_{*}(\cdot) \equiv \boldsymbol{T}_{i^{*}(\boldsymbol{W}(\cdot), \boldsymbol{R}(\cdot))}(\cdot)$ and $\boldsymbol{W}_{*}(\cdot) \equiv \boldsymbol{W}_{i^{*}(\boldsymbol{W}(\cdot), \boldsymbol{R}(\cdot))}(\cdot)$.

Remark 3: At any $t \in \mathbb{Z}_{+}$, the state, $\boldsymbol{S}(t)$, of the system given by,

$$
\begin{aligned}
\boldsymbol{S}(t) \equiv(\boldsymbol{Q}(t) ; \boldsymbol{W}(t) & =\left(\boldsymbol{W}_{i}(t), 1 \leq i \leq \boldsymbol{Q}(t)\right) ; \\
\boldsymbol{L}(t) & \left.=\left(\boldsymbol{L}_{i}(t), 1 \leq i \leq \boldsymbol{Q}(t)\right)\right),
\end{aligned}
$$

forms a discrete time homogeneous Markov chain under the MaxWeight scheduler.

\section{MAIN RESULT}

The main result of this paper is given below and in the rest of the paper we will sketch the proof of this result.

Theorem 1: Delay-driven MaxWeight scheduler is throughput optimal, i.e., for any $\rho<1$ in the system described in Section II, the delay-driven MaxWeight scheduler stabilizes the system.

We will need the following quantities all of which are derived from a system samplepath $^{2} \mathcal{X} \equiv(S(t), A(t), R(t), t \in \mathbb{Z})$.

- Let $U(t) \equiv\left(U_{i}(t), 1 \leq i \leq Q(t)\right)$, where $U_{i}(t) \equiv\left\lceil\frac{L_{i}(t)}{r^{\max }}\right\rceil$, i.e., the number of slots it will take to serve the $i^{\text {th }}$ present file if served at rate $r^{\max }$ bits/slots.

\footnotetext{
${ }^{2}$ Even though $(A(t), t \geq 0)$ can be inferred from the other components of $\mathcal{X}$, for clarity we explicitly include $(A(t), t \geq 0)$ in definition of $\mathcal{X}$.
} 


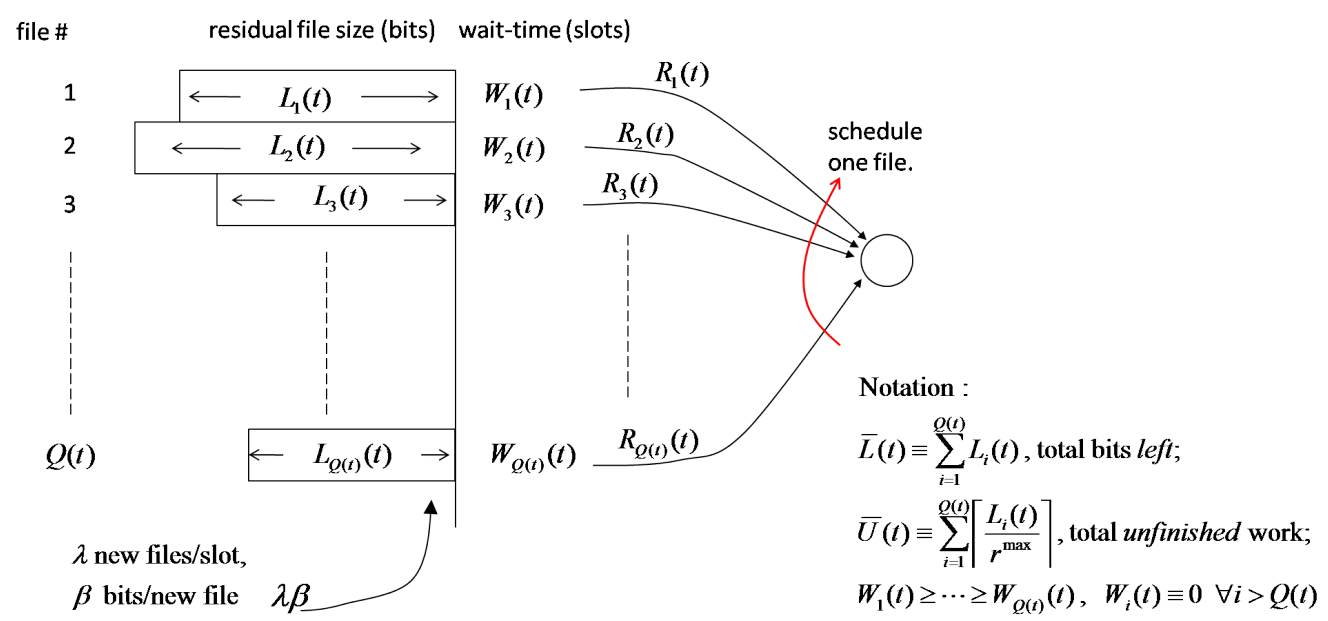

Fig. 1. System model.

- Let $\bar{U}(t) \equiv \sum_{i=1}^{Q(t)} U_{i}(t)$, i.e., the total unfinished work present in the system (assuming the service rate is always $\left.r^{\max }\right)$ at the end of slot $[t-1, t)$.

- Let $\bar{L}(t) \equiv \sum_{i=1}^{Q(t)} L_{i}(t)$, i.e., the total number of bits present at the end of slot $[t-1, t)$.

In the sequel, we will extend the domain of all discrete time processes and functions to continuous time: a function originally defined on integer times has the same value at any real $t$ that it takes at $\lfloor t\rfloor$. Also, we will extend the definition of $W_{i}(t)$ to all $i \in\{1,2, \ldots\}$ by letting $W_{i}(t)=0$ for $i \in\{Q(t)+1, Q(t)+2, \cdots\}$.

For each $n \in \mathbb{Z}_{+}$, consider a independent and stochastically equivalent system

$$
\boldsymbol{\mathcal { X }}^{(n)} \equiv\left(S^{(n)}(0) ; \boldsymbol{S}^{(n)}(t+1), \boldsymbol{A}^{(n)}(t), \boldsymbol{R}^{(n)}(t), t \geq 0\right)
$$

under the delay-driven MaxWeight scheduler and where the initial state $S^{(n)}(0)$ is non-random and satisfies,

$$
\left\|S^{(n)}(0)\right\| \equiv \bar{U}^{(n)}(0)+W_{1}^{(n)}(0)=n,
$$

i.e., the total work in terms of number of slots it will take to serve the files present at $t=0$ if served at rate $r^{\max }$ per slot, plus the wait time of the oldest file in the system.

The following proposition is due to [12], and will be used in proving Theorem 1.

Proposition 1: Suppose there exists an $\epsilon>0$ and an integer $t_{1}>0$ such that the following holds for any sequence of systems $\left\{\mathcal{X}^{(n)}, n=1,2, \ldots\right\}$ satisfying (2),

$$
\limsup _{n \rightarrow \infty} \mathbb{E}\left(\frac{1}{n}\left\|\boldsymbol{S}^{(n)}\left(n t_{1}\right)\right\|\right) \leq 1-\epsilon,
$$

then the Markov chain $(\boldsymbol{S}(t), t \in \mathbb{Z})$ is stable.

See [7] for a use of this proposition to show throughput optimality of queue- and delay-driven MaxWeight in the case of a system with a fixed number of flows each having a corresponding stationary exogenous packet arrival process.
We will now define the fluid-scaled functions and processes obtained from the sequence $\left\{\boldsymbol{\mathcal { X }}^{(n)}, n=1,2, \ldots\right\}$; these fluidscaled functions and their limits will be used in showing the conditions required for Proposition 1, i.e., the existence of $\epsilon$ and $t_{1}$ as in (3).

1) Fluid limit of the deterministic initial state: For $t \in(-\infty, 0]$, let $F^{(n)}(t)$ denote the number of files present at time 0 that had arrived by the end of time slot $[\lfloor t\rfloor-1,\lfloor t\rfloor)$, i.e.,

$$
F^{(n)}(t) \equiv \sum_{i=1}^{Q^{(n)}(0)} \mathbb{1}_{\left\{T_{i}^{(n)}(0) \leq t-1\right\}} .
$$

Since $W_{1}^{(n)}(0) \leq n$, we have that $F^{(n)}(-n)=0$ and $F^{(n)}(0)=Q^{(n)}(t)$.

Let,

$$
\begin{aligned}
\bar{l}^{(n)}(0) & \equiv \frac{1}{n} \bar{L}^{(n)}(0), \\
\bar{u}^{(n)}(0) & \equiv \frac{1}{n} \bar{U}^{(n)}(0), \\
q^{(n)}(0) & \equiv \frac{1}{n} Q^{(n)}(0),
\end{aligned}
$$

and for $t \in[-1,0]$, let,

$$
f^{(n)}(t) \equiv \frac{1}{n} F^{(n)}(n t),
$$

and for $x \in[0, \infty)$, let ${ }^{3}$,

$$
w_{x}^{(n)}(0) \equiv \frac{1}{n} W_{\lfloor n x\rfloor+1}^{(n)}(0),
$$

where $w_{x}^{(n)}(0)$ is decreasing in $x$. Since,

$$
0 \leq q^{(n)}(0) \leq \bar{l}^{(n)}(0) \leq r^{\max }\left(\bar{u}^{(n)}(0)+w_{0}^{(n)}(0)\right)=r^{\max },
$$

${ }^{3}\left(f^{(n)}(t),-1 \leq t \leq 0\right)$ and $\left(w_{x}^{(n)}(0), 0 \leq x \leq q^{(n)}(0)\right)$ are related. That is, $w_{x}^{(n)}(0)=1 / n-\inf _{t}\left(t: f^{(n)}(t) \geq x+1 / n\right)$. 
therefore, along some subsequence of $n$ (for simplicity still denoted by $n$ ), we have,

$$
\begin{aligned}
\bar{l}^{(n)}(0) & \rightarrow \bar{l}(0), & \bar{u}^{(n)}(0) & \rightarrow \bar{u}(0), \\
w_{0}^{(n)}(0) & \rightarrow w_{0}(0), & q^{(n)}(0) & \rightarrow q(0),
\end{aligned}
$$

where $\bar{u}(0)+w_{0}(0)=1$. With $w_{0}(0)$ and $f(0) \equiv q(0)$ as above, let $\left(w_{x}(0), 0 \leq x \leq q(0)\right)$ and $(f(t),-1 \leq t \leq 0)$ be weak limits of $\left(w_{x}^{(n)}(0), 0 \leq x \leq q(0)\right)$ and $\left(f^{(n)}(t), \quad-1 \leq x \leq 0\right)$ respectively along a further subsequence of $n$, i.e.,

$$
w_{x}^{(n)}(0) \rightarrow w_{x}(0), \quad f^{(n)}(t) \rightarrow f(t)
$$

at the points of continuity of $w_{(\cdot)}(0)$ and $f(\cdot)$ respectively. To summarize, the (partial) initial state of the limiting system is captured by,

$$
\begin{aligned}
(q(0) ; & w(0) \equiv\left(w_{x}(0), 0 \leq x \leq q(0)\right) \\
& \left.(f(t), \quad-1 \leq t \leq 0) ; \bar{u}(0)+w_{0}(0)=1\right) .
\end{aligned}
$$

2) Deterministic fluid limit of the random state for $t \geq 0$ : We will also need the following fluid-scaled processes, all defined for $t \in \mathbb{R}_{+}$.

Let $\boldsymbol{F}^{(n)}(t), \boldsymbol{F}_{l}^{(n)}(t)$, and $\boldsymbol{F}_{u}^{(n)}(t)$ respectively be the total files, the total bits, and the total work arriving up to the end of time slot $[\lfloor t\rfloor-1,[\lfloor t\rfloor)$, i.e.,

$$
\begin{aligned}
\boldsymbol{F}^{(n)}(t) & \equiv Q^{(n)}(0)+\sum_{k=0}^{t-1} \boldsymbol{A}^{(n)}(k), \\
\boldsymbol{F}_{l}^{(n)}(t) & \equiv \bar{L}^{(n)}(0)+\sum_{k=0}^{t-1} \sum_{m=1}^{\boldsymbol{A}(k)} \boldsymbol{B}_{m}^{(n)}(k), \\
\boldsymbol{F}_{u}^{(n)}(t) & \equiv \bar{U}^{(n)}(0)+\sum_{k=0}^{t-1} \sum_{m=1}^{\boldsymbol{A}(k)}\left\lceil\frac{\boldsymbol{B}_{m}^{(n)}(k)}{r^{\max }}\right\rceil .
\end{aligned}
$$

Then, along a further subsequence of $n$, we have the following uniform over compact sets (u.o.c) convergences (see Theorem 4.1 of [13], Lemma 1 of [7]),

$$
\begin{aligned}
&\left(\boldsymbol{f}^{(n)}(t)\right.\left.\equiv \frac{1}{n} \boldsymbol{F}^{(n)}(n t), t \geq 0\right) \\
&(f(t) \equiv q(0)+\lambda t, t \geq 0), \\
&\left(\boldsymbol{f}_{l}^{(n)}(t) \equiv \frac{1}{n} \boldsymbol{F}_{l}^{(n)}(n t), t \geq 0\right) \rightarrow \\
&\left(f_{l}(t)\right.\equiv \bar{l}(0)+\lambda \beta t, t \geq 0), \\
&\left(\boldsymbol{f}_{u}^{(n)}(t) \equiv \frac{1}{n} \boldsymbol{F}_{u}^{(n)}(n t), t \geq 0\right) \rightarrow \\
&\left(f_{u}(t)\right.\equiv \bar{u}(0)+\rho t, t \geq 0) .
\end{aligned}
$$

Let $\boldsymbol{D}^{(n)}(\tau, t), \boldsymbol{D}_{l}^{(n)}(\tau, t)$, and $\boldsymbol{D}_{u}^{(n)}(\tau, t)$ respectively be the total files, the total bits, and the total work which

(i) arrived before the end of time slot $[\lfloor\tau\rfloor-1,\lfloor\tau\rfloor$ ), and

(ii) departed/completed service by the end of time slot $[\lfloor t\rfloor-1,\lfloor t\rfloor)$.
These can be mathematically defined as follows: for $t \geq 0, t \geq \tau>-\infty$,

$$
\begin{aligned}
& \boldsymbol{D}^{(n)}(\tau, t) \equiv \sum_{k=0}^{t-1} \mathbb{1}_{\left\{\boldsymbol{L}_{*}^{(n)}(k) \leq \boldsymbol{R}_{*}^{(n)}(k), \boldsymbol{T}_{*}^{(n)}(k) \leq \tau-1\right\}}, \\
& \boldsymbol{D}_{l}^{(n)}(\tau, t) \equiv \sum_{k=0}^{t-1} \min \left(\boldsymbol{L}_{*}^{(n)}(k), \boldsymbol{R}_{*}^{(n)}(k)\right) \mathbb{1}_{\left\{\boldsymbol{T}_{*}^{(n)}(k) \leq \tau-1\right\}}, \\
& \boldsymbol{D}_{u}^{(n)}(\tau, t) \equiv \sum_{k=0}^{t-1}\left(\left\lceil\frac{\boldsymbol{L}_{*}^{(n)}(k)}{r^{\max }}\right\rceil-\left\lceil\frac{\boldsymbol{L}_{*}^{(n)}(k)-\boldsymbol{R}_{*}^{(n)}(k)}{r^{\max }}\right\rceil\right) \times \\
& \mathbb{1}_{\left\{\boldsymbol{T}_{*}^{(n)}(k) \leq \tau-1\right\}} .
\end{aligned}
$$

For example, $\boldsymbol{D}^{(n)}(t, t)$ is simply the total number of files which have completed service by the end of slot $[\lfloor t\rfloor-1,\lfloor t\rfloor)$. Moreover, $\boldsymbol{D}^{(n)}(\tau, t)$ is increasing in $\tau$ and $t$ with $\boldsymbol{D}^{(n)}(\cdot, 0)=0$, and for any $\left(\tau_{1}, t_{1}\right) \leq\left(\tau_{2}, t_{2}\right)$, we have that $\boldsymbol{D}^{(n)}\left(\tau_{2}, t_{2}\right)-\boldsymbol{D}^{(n)}\left(\tau_{1}, t_{1}\right) \leq \max \left(t_{2}-t_{1}+\right.$ $\left.1, \boldsymbol{F}^{(n)}\left(\tau_{2}\right)-\boldsymbol{F}^{(n)}\left(\tau_{1}\right)\right)$. Similar bounds hold for $\boldsymbol{D}_{l}^{(n)}(\tau, t)$ and $\boldsymbol{D}_{u}^{(n)}(\tau, t)$. Then, along a further subsequence of $n$, we have the following u.o.c. convergences to Lipschitz continuous (and hence differentiable a.e.) limiting functions,

$$
\begin{aligned}
\left(\boldsymbol{d}^{(n)}(\tau, t)=\frac{1}{n} \boldsymbol{D}^{(n)}(n \tau, n t),\right. & t \geq \tau \geq 0) \rightarrow \\
& (d(\tau, t), t \geq \tau \geq 0) \\
\left(\boldsymbol{d}_{l}^{(n)}(\tau, t) \equiv \frac{1}{n} \boldsymbol{D}_{l}^{(n)}(n \tau, n t),\right. & t \geq \tau \geq 0) \rightarrow \\
& \left(d_{l}(\tau, t), t \geq \tau \geq 0\right), \\
\left(\boldsymbol{d}_{u}^{(n)}(\tau, t) \equiv \frac{1}{n} \boldsymbol{D}_{u}^{(n)}(n \tau, n t),\right. & t \geq \tau \geq 0) \rightarrow \\
& \left(d_{u}(\tau, t), t \geq \tau \geq 0\right) .
\end{aligned}
$$

The points $(\tau, t)$ where the derivatives of the limiting functions exist are called regular. Then, for all regular $t \geq \tau \geq 0$, we have that $0 \leq \frac{\partial d(\tau, t)}{\partial \tau} \leq \lambda$ and $0 \leq \frac{\partial d(\tau, t)}{\partial t} \leq 1$. Similar bounds hold for the derivatives of $d_{l}(\tau, t)$ and $d_{u}(\tau, t)$.

Let $\boldsymbol{Q}^{(n)}(\tau, t)$ denote the number of files which arrived before the end of slot $[\tau-1, \tau)$ but are still present at the end of slot $[t-1, t)$; so, for example, $\boldsymbol{Q}^{(n)}(t)=\boldsymbol{Q}^{(n)}(t, t)$. Then using the above defined processes, we have that,

$$
\begin{aligned}
\boldsymbol{Q}^{(n)}(\tau, t) & =\boldsymbol{F}^{(n)}(\tau)-\boldsymbol{D}^{(n)}(\tau, t), \\
\boldsymbol{Q}^{(n)}(t) & =\boldsymbol{Q}^{(n)}(t, t)=\boldsymbol{F}^{(n)}(t)-\boldsymbol{D}^{(n)}(t, t), \\
\overline{\boldsymbol{L}}^{(n)}(t) & =\boldsymbol{F}_{l}^{(n)}(t)-\boldsymbol{D}_{l}^{(n)}(t, t), \\
\overline{\boldsymbol{U}}^{(n)}(t) & =\boldsymbol{F}_{u}^{(n)}(t)-\boldsymbol{D}_{u}^{(n)}(t, t), \\
\boldsymbol{W}_{i}^{(n)}(t) & =\lfloor t\rfloor+1-\inf _{\tau}\left(\tau: \boldsymbol{Q}^{(n)}(\tau, t) \geq i\right), \\
& \quad i \in\left\{1, \cdots, \boldsymbol{Q}^{(n)}(t)\right\},
\end{aligned}
$$

and the following u.o.c. convergences to Lipschitz continuous 
limiting functions,

$$
\begin{aligned}
\left(\boldsymbol{q}^{(n)}(\tau, t) \equiv\right. & \left.\frac{1}{n} \boldsymbol{Q}^{(n)}(n \tau, n t), t \geq \tau \geq 0\right) \rightarrow \\
& (q(\tau, t) \equiv f(\tau)-d(\tau, t), t \geq \tau \geq 0) \\
\left(\boldsymbol{q}^{(n)}(t) \equiv\right. & \left.\frac{1}{n} \boldsymbol{Q}^{(n)}(n t), t \geq 0\right) \rightarrow \\
& (q(t) \equiv f(t)-d(t, t), t \geq 0) \\
\left(\overline{\boldsymbol{l}}^{(n)}(t) \equiv\right. & \left.\frac{1}{n} \overline{\boldsymbol{L}}^{(n)}(n t), t \geq 0\right) \rightarrow \\
& \left(\bar{l}(t) \equiv f_{l}(t)-d_{l}(t, t), t \geq 0\right) \\
\left(\overline{\boldsymbol{u}}^{(n)}(t) \equiv\right. & \left.\frac{1}{n} \overline{\boldsymbol{U}}^{(n)}(n t), t \geq 0\right) \rightarrow \\
& \left(\bar{u}(t) \equiv f_{u}(t)-d_{u}(t, t), t \geq 0\right)
\end{aligned}
$$

and the following weak convergence,

$$
\begin{aligned}
\left(\boldsymbol{w}_{x}^{(n)}(t) \equiv \frac{1}{n} \boldsymbol{W}_{\lfloor n x\rfloor+1}^{(n)}(n t),\right. & x \geq 0, t \geq 0) \rightarrow \\
& \left(w_{x}(t), x \geq 0, t \geq 0\right),
\end{aligned}
$$

where, if $q(t)>0=q(0, t)$, then $w_{0}(t)$ is given by the rightlimit of $w_{x}(t)$, i.e.,

$$
\begin{aligned}
w_{0}(t)=\lim _{x \downarrow 0} w_{x}(t) & =\lim _{x \downarrow 0}\left(t-\inf _{\tau}(\tau: q(\tau, t) \geq x)\right), \\
& =t-\sup _{\tau}(\tau: q(\tau, t)=0) .
\end{aligned}
$$

3) Dynamics and derivatives of fluid limit for $t \geq 0$ : The limiting point $\left(q, \bar{l}, \bar{u}, w, f, f_{l}, f_{u}, d, d_{l}, d_{u}\right)$ of the scaled version of sequence $\left\{\mathcal{X}^{(n)}, n=1,2, \ldots\right\}$ obtained above is not necessarily unique. In particular, the limit point depends on the sequence of initial states $\left\{S^{(n)}(0)\right\}$ and the convergent subsequence chosen. However, the following lemmas hold for all limit points of the scaled version of sequence $\left\{\boldsymbol{\mathcal { X }}^{(n)}, n=1,2, \ldots\right\}$. See Appendix for proofs.

Lemma 1: Consider any set of limiting functions derived from $\left\{\mathcal{X}^{(n)}, n=1,2, \ldots\right\}$. For any regular $t \geq \tau \geq 0$, if $f_{l}(\tau)-d_{l}(\tau, t)>0$, then,

$$
\frac{\partial d_{l}(\tau, t)}{\partial t} \geq 1
$$

and,

$$
\frac{\partial d_{l}(t, t)}{\partial t}=\frac{\partial d_{l}(\tau, t)}{\partial t}
$$

Remark 4: The condition $f_{l}(\tau)-d_{l}(\tau, t)>0$ means that there is a strictly positive amount of bit fluid with wait time at least $t-\tau$. Then (6) ensures that this fluid will eventually get served, whereas, (7) ensures no service is given to any newer fluid while the older one remains in the system. This lemma however does not establish that the rate at which the fluid is being served is higher than the rate at which the fluid is entering the system; that is addressed later in Lemma 2.
Corollary 1: By (4) and (6) in Lemma 1, for all $t>T_{0} \equiv r^{\max }$, we have,

$$
w_{0}(t)<t,
$$

i.e., all the fluid initially present in the system (recall $\bar{l}(0)$ ) gets served by time $T_{0}$. Moreover, by (7) in Lemma 1, the fluid is served in a FCFS (first-come-first-serve) manner, therefore, for all $t>T_{0}$, we have that,

$$
\begin{aligned}
w_{x}(t) & =\frac{q(t)-x}{\lambda}, \quad x \in[0, q(t)] ; \\
\bar{l}(t) & =\beta q(t) ; \\
\bar{u}(t) & =\gamma q(t) .
\end{aligned}
$$

Remark 5: To see (8), pick an $x \in(0, q(t))$ and note that $\tau \equiv t-w_{x}(t) \geq t-w_{0}(t)>0$ and $q(\tau, t) \geq x>0$. Then $f(t)-f(\tau)=\lambda w_{x}(t)$. By Lemma 1, this $\lambda w_{x}(t)$ amount of file fluid that has entered the system since $\tau$ must still be queued behind $x$. That is, $q(t)-x=\lambda w_{x}(t)$. Equations (9) and (10) follow similarly.

Lemma 2: Consider any set of limiting functions derived from $\left\{\mathcal{X}^{(n)}, n=1,2, \ldots\right\}$. For any regular $t>T_{0}$, if $q(t)>0$, then,

$$
\frac{\partial d(t, t)}{\partial t}=\gamma^{-1}, \quad \frac{\partial d_{u}(t, t)}{\partial t}=1
$$

and hence,

$$
\begin{aligned}
q^{\prime}(t) & =\lambda-\gamma^{-1}<0, \\
\bar{u}^{\prime}(t) & =\rho-1<0, \\
w_{0}^{\prime}(t) & =\frac{q^{\prime}(t)}{\lambda}<0 .
\end{aligned}
$$

Corollary 2: There exits a finite $T_{1}$ (independent of the set $\left.\left\{\mathcal{X}^{(n)}, n=1,2, \ldots\right\}\right)$, such that for any set of limiting functions and for all $t>T_{1}$, we have that

$$
\bar{u}(t)+w_{0}(t)=0 \text {. }
$$

4) Using Proposition 1 to conclude the proof of Theorem 1: Corollary 2 implies that,

$$
\lim _{n \rightarrow \infty} \frac{1}{n}\left\|\boldsymbol{S}^{(n)}\left(n T_{1}\right)\right\|=0 \text { a.s. }
$$

Moreover, the sequence of random variables $\left\{\frac{1}{n}\left\|\boldsymbol{S}^{(n)}\left(n T_{1}\right)\right\|, n=1,2, \ldots\right\} \quad$ is uniformly integrable since (see [14], p. 351),

$$
\begin{aligned}
\frac{1}{n}\left\|\boldsymbol{S}^{(n)}\left(n T_{1}\right)\right\| & \leq 1+\boldsymbol{f}_{u}^{(n)}\left(T_{1}\right)+T_{1} \stackrel{\text { a.s. }}{\longrightarrow} \\
& \mathbb{E}\left(1+\boldsymbol{f}_{u}^{(n)}\left(T_{1}\right)+T_{1}\right)=1+(\rho+1) T_{1}<\infty .
\end{aligned}
$$

Then, the almost sure convergence in (12) along with uniform integrability implies the following convergence in the mean,

$$
\lim _{n \rightarrow \infty} \mathbb{E}\left(\frac{1}{n}\left\|\boldsymbol{S}^{(n)}\left(n T_{1}\right)\right\|\right)=0,
$$

thus completing the proof of throughput optimality of the delay-driven MaxWeight. 


\section{CONCLUSION AND EXTENSIONS}

An interesting extension of the system model is to allow that a file may arrive gradually over time instead of all at once (i.e., similar to the case (ii) of [5]). Assuming the distribution of the time interval over which the first and the last bit of a file arrives has a light tail, the extension of the current result seems possible without much effort: the fluid limits of the arrival process (e.g., the work arrival process $f_{u}$ ) is indistinguishable for the two cases. Similarly, the assumption that channel $\boldsymbol{R}_{i}(t)$ be i.i.d. over time can be relaxed to, e.g., $\boldsymbol{R}_{i}(t)$ forming a Markov chain in the set $\left\{0, \cdots, r^{\max }\right\}$ with a unique stationary distribution satisfying $p=\mathbb{P}\left(\boldsymbol{R}_{i}(\cdot)=r^{\max }\right)>0$, and $\boldsymbol{R}_{i}(\cdot)$ being drawn from the stationary distribution upon the arrival of a file. Other interesting extensions that seem possible, however, require little more effort are as follows: allowing for different classes to have a possibly different $r^{\max }$ bits/slot, i.e., the maximum rate (with non-zero probability) supported by the channel; and generalizing the scheduler to weighted-delay-driven MaxWeight, where the wait time of each file is scaled by a fixed, class-dependant constant (see (1)).

\section{APPENDIX}

Proof of Lemma 1: Fix a $t \geq \tau \geq 0$ such that $f_{l}(\tau)-d_{l}(\tau, t)>0$. Then by the assumption of bounded file size, we have $q(\tau, t)>0$. Since $q(\tau, \cdot)$ is Lipschitz continuous, there exits a $\delta>0$ and $0 \leq t_{1}<t_{2}$ such that $t \in\left[t_{1}, t_{2}\right]$ and for all $\hat{t} \in\left[t_{1}, t_{2}\right]$ we have $q(\tau, \hat{t})>2 \delta$. By uniform convergence of $q^{(n)}(\tau, \cdot) \rightarrow q(\tau, \cdot)$ over $\left[t_{1}, t_{2}\right]$, for all large $n$ we have that over $\left[t_{1}, t_{2}\right], \boldsymbol{q}^{(n)}(\tau, \cdot)>\delta$ and $\boldsymbol{w}_{x}^{(n)}(\cdot) \geq(\tau-\cdot)$ for $x \in[0, \delta]$. Finally,

$$
\begin{aligned}
t_{2} & -t_{1}+\frac{1}{n} \\
\geq & \frac{1}{n} \sum_{k=n t_{1}}^{n t_{2}-1} \mathbb{1}_{\left\{i_{*}\left(\boldsymbol{W}^{(n)}(k), \boldsymbol{R}^{(n)}(k)\right) \leq n \delta\right\}}, \\
\geq & \frac{1}{n} \sum_{k=n t_{1}}^{n t_{2}-1} \mathbb{1}_{\left\{\max _{1 \leq i \leq n \delta} \boldsymbol{W}_{i}^{(n)}(k) \boldsymbol{R}_{i}^{(n)}(k) \geq(n \tau-k) r_{\max }\right\}}, \\
\geq & \frac{1}{n} \sum_{k=n t_{1}}^{n t_{2}-1} \mathbb{1}_{\left\{\max _{1 \leq i \leq n \delta} \boldsymbol{R}_{i}^{(n)}(k)=r^{\max }\right\}} \stackrel{a . s .}{\longrightarrow} t_{2}-t_{1} .
\end{aligned}
$$

That is, $\liminf _{n \rightarrow \infty} \boldsymbol{d}_{l}^{(n)}\left(\tau, t_{2}\right)-\boldsymbol{d}_{l}^{(n)}\left(\tau, t_{1}\right) \geq t_{2}-t_{1}$ a.s. and $\lim _{n \rightarrow \infty}\left(\boldsymbol{d}_{l}^{(n)}\left(t_{2}, t_{2}\right)-\boldsymbol{d}_{l}^{(n)}\left(t_{1}, t_{1}\right)\right)-\left(\boldsymbol{d}_{l}^{(n)}\left(\tau, t_{2}\right)-\right.$ $\left.\boldsymbol{d}_{l}^{(n)}\left(\tau, t_{1}\right)\right)=0$ a.s.

Proof of Lemma 2: Fix a $t>T_{0}$ such that $q(t)>0$. Since $\boldsymbol{w}_{(\cdot)}^{(n)}\left(T_{0}+\cdot\right)$ converges u.o.c. to Lipschitz continuous $w_{(\cdot)}(\cdot)$ (see (8)), we can pick a $q(t)>\delta>0$ and $T_{0} \leq t_{1}<t_{2}$ such that $t \in\left[t_{1}, t_{2}\right]$ and for all large $n$ uniformly over $\left[t_{1}, t_{2}\right]$, we have $r^{\max } \boldsymbol{w}_{\delta}^{(n)}(\cdot) \geq\left(r^{\max }-1\right) \boldsymbol{w}_{0}^{(n)}(\cdot)$. This choice of $\delta$ implies that for any $\hat{t} \in\left\{\left\lfloor n t_{1}\right\rfloor, \cdots,\left\lfloor n t_{2}\right\rfloor-1\right\}$, if a file in set $\{1, \cdots,\lfloor n \delta\rfloor\}$ sees a channel state of $r^{\max }$, then $\boldsymbol{R}_{*}(\hat{t})=r^{\max }$. Rest of the proof proceeds similar to that of
Lemma 1, except that we will strengthen the event associated with the Indicator function in (13). We have that,

$$
\begin{aligned}
t_{2} & -t_{1}+\frac{1}{n} \\
& \geq \frac{1}{n} \sum_{k=n t_{1}}^{n t_{2}-1} \mathbb{1}_{\left\{\boldsymbol{R}_{*}^{(n)}(k)=r_{\max }\right\}} \\
& \geq \frac{1}{n} \sum_{k=n t_{1}}^{n t_{2}-1} \mathbb{1}_{\left\{i^{*}\left(\boldsymbol{W}^{(n)}(k), \boldsymbol{R}^{(n)}(k)\right) \leq n \delta, \boldsymbol{R}_{*}^{(n)}(k)=r^{\max }\right\}}, \\
& =\frac{1}{n} \sum_{k=n t_{1}}^{n t_{2}-1} \mathbb{1}_{\left\{\max _{1 \leq i \leq n \delta} \boldsymbol{R}_{i}^{(n)}(k)=r^{\max }\right\}}, \\
& \geq \frac{1}{n} \sum_{k=n t_{1}}^{n t_{2}-1} \mathbb{1}_{\left\{\max _{1 \leq i \leq n \delta} \boldsymbol{R}_{i}^{(n)}(k) \geq r^{\max }\right\}}^{\stackrel{a . s .}{\longrightarrow} t_{2}-t_{1} .}
\end{aligned}
$$

Then, along with Corollary 1, it follows that $\lim _{n \rightarrow \infty} \boldsymbol{d}_{u}^{(n)}\left(t_{2}, t_{2}\right)-\boldsymbol{d}_{u}^{(n)}\left(t_{1}, t_{1}\right)=t_{2}-t_{1} \quad$ a.s. and $\lim _{n \rightarrow \infty} \boldsymbol{d}^{(n)}\left(t_{2}, t_{2}\right)-\boldsymbol{d}^{(n)}\left(t_{1}, t_{1}\right)=\gamma^{-1}\left(t_{2}-t_{1}\right)$ a.s.

\section{REFERENCES}

[1] R. Knopp and P. Humblet, "Information capacity and power control in single-cell multiuser communications," in Proc. IEEE Int. Conf. on Comm., vol. 1, Jun 1995, pp. 331-335.

[2] P. Viswanath, D. Tse, and R. Laroia, "Opportunistic beamforming using dumb antennas," IEEE Trans. Infomation Theory, vol. 48, no. 6, pp. 1277-1294, Jun 2002.

[3] X. Liu, E. K. P. Chong, and N. B. Shroff, "A framework for opportunistic scheduling in wireless networks," Comput. Networks, vol. 41, no. 4, pp. 451-474, 2003.

[4] M. Andrews, "Instability of the proportional fair scheduling algorithm for HDR,” IEEE Trans. Wireless Comm., vol. 3, no. 5, pp. 1422-1426, Sept. 2004.

[5] P. van de Ven, S. Borst, and S. Shneer, "Instability of maxweight scheduling algorithms," in Proc. IEEE INFOCOM, April 2009, pp. 1701-1709.

[6] L. Tassiulas and A. Ephremides, "Dynamic server allocation to parallel queues with randomly varying connectivity," IEEE Trans. Infomation Theory, vol. 39, no. 2, pp. 466-478, Mar 1993.

[7] M. Andrews, K. Kumaran, K. Ramanan, A. Stolyar, R. Vijayakumar, and P. Whiting, "Scheduling in a queuing system with asynchronously varying service rates," Probab. Eng. Inf. Sci., vol. 18, no. 2, pp. 191-217, 2004.

[8] S. Shakkottai and A. Stolyar, "Scheduling for multiple flows sharing a time-varying channel: The Exponential Rule," American Mathematical Society Translations, Series 2, vol. 207, 2002.

[9] B. Sadiq, S. Baek, and G. de Veciana, "Delay-optimal opportunistic scheduling and approximations: the Log rule," in Proc. IEEE INFOCOM, Rio de Janeiro, Brazil, 2009.

[10] S. Shakkottai and A. Stolyar, "Scheduling algorithms for a mixture of real-time and non-real-time data in HDR," in Proceedings of the 17th International Teletraffic Congress (ITC-17), 2001.

[11] B. Sadiq, R. Madan, and A. Sampath, "Downlink scheduling for multiclass traffic in LTE," EURASIP Jour. Wireless Comm. Net., Aug 2009.

[12] V. A. Malyshev and M. V. Menshikov, "Ergodicity, continuity, and analyticity of countable markov chains," Transactions of Moscow Mathematical Society, 1979.

[13] J. G. Dai, "On positive harris recurrence of multiclass queueing networks: A unified approach via fluid limit models," Annals of Applied Probability, vol. 5, pp. 49-77, 1995.

[14] G. Grimmett and D. Stirzaker, Probability and Random Processes, 3rd ed. Oxford, 2001. 